\title{
Passive Time Reversal Acoustic Communications Through Shallow-Water Internal Waves
}

\author{
Aijun Song, Member, IEEE, Mohsen Badiey, Member, IEEE, Arthur E. Newhall, Member, IEEE, \\ James F. Lynch, Fellow, IEEE, Harry A. DeFerrari, and Boris G. Katsnelson
}

\begin{abstract}
During a 12-h period in the 2006 Shallow Water Experiment (SW06), binary phase shift keying (BPSK) signals at the carrier frequencies of 813 and $1627 \mathrm{~Hz}$ were propagated over a 19.8-km source-receiver range when a packet of strong internal waves passed through the acoustic track. The communication data are analyzed by time reversal processing followed by a single-channel decision feedback equalizer. Two types of internal wave effects are investigated in the context of acoustic communications. One is the rapid channel fluctuation within 90-s data packets. It can be characterized as decreased channel coherence, which was the result of fast sound-speed perturbations during the internal wave passage. We show its effect on the time reversal receiver performance and apply channel tracking in the receiver to counteract such fluctuation. The other one is the long-term (in the scale of hours) performance degradation in the depressed waveguide when the internal waves passed through the acoustic track. Even with channel tracking, the time reversal receiver experiences average 3-4-dB decrease in the output signal-to-noise ratio (SNR). Such long-term performance degradation is explained by the ray approximation in the depressed waveguide.
\end{abstract}

Index Terms-Acoustic communications, decision feedback equalizers, internal waves, time reversal processing.

\section{INTRODUCTION}

$\mathbf{U}$ NDERWATER acoustic channels are challenging for digital communications due to excessive multipath spread [1]. Further, the variability of the ocean environment can cause fluctuations of acoustic channels that can result in additional limitations on digital communications [2]-[4]. Among the dynamic ocean processes, internal waves can cause significant variation of acoustic pulses propagating in shallow water [5]. There have been a number of experimental and modeling efforts to investigate acoustic fluctuations in the presence of internal

Manuscript received February 03, 2009; revised December 23, 2009, March 17, 2010, and June 25, 2010; accepted June 26, 2010. Date of publication November 09, 2010; date of current version November 30, 2010. This work was supported by the U.S. Office of Naval Research (ONR) Code 322OA under Grants N00014-07-1-0546 and N00014-06-1019.

Associate Editor: H. Song.

A. Song and M. Badiey are with the College of Earth, Ocean, and Environment, University of Delaware, Newark, DE 19716 USA (e-mail: ajsong@udel. edu).

A. E. Newhall and J. F. Lynch are with the Department of Applied Ocean Physics and Engineering, Woods Hole Oceanographic Institution, Woods Hole, MA 02543 USA.

H. A. DeFerrari is with the Division of Applied Marine Physics, University of Miami, Miami, FL 33149 USA.

Boris G. Katsnelson is with the Physics Department, Voronezh State University, Voronezh 394006, Russia.

Color versions of one or more of the figures in this paper are available online at http://ieeexplore.ieee.org.

Digital Object Identifier 10.1109/JOE.2010.2060530 waves. For example, internal waves were reported causing intensity fluctuations of acoustic pulses during the 1995 Shallow Water Acoustics in Random Media (SWARM-95) Experiment [6] and these fluctuations were later modeled [7], [8]. The coherence properties of acoustic pulses in an internal wave field have also been studied [9]-[12]. Both coherence and intensity fluctuations of the acoustic channel can affect the performance of coherent underwater acoustic communications. However, only limited efforts have been reported to relate these effects to the performance of acoustic communications. The only notable example is [13], where signal temporal coherence in several experiments has been summarized. Sample demodulation results at the carrier frequency of $400 \mathrm{~Hz}$ have been used to show effects of the decreased signal coherence on the decision feedback equalizer (DFE). The decrease of signal coherence has been attributed to the presence of internal waves, which were referred to as the common physical processes in the experimental sites.

Time reversal is a pulse compression technique which focuses on sound transmissions that have been spread by propagation in a multipath environment without prior knowledge of the medium [14]. After being first demonstrated in the 1990s [15], both active [16] and passive [17] time reversals have been investigated for acoustic communications. More recently, the time reversal approach has been combined with DFEs to suppress the residual intersymbol interference (ISI) [18], [19] for carrier frequencies of 3-5 kHz or below. Channel and phase tracking has also been incorporated into time reversal receivers to achieve reliable communications in dynamic ocean environments at a higher central frequency $(12 \mathrm{kHz})[4]$.

During the 2006 Shallow Water Experiment (SW06) [20], concurrent environmental observations and acoustic measurements were made to study internal wave effects on coherent underwater acoustic communications. During a 12-h communication period over a 19.8-km acoustic track, a packet of strong internal waves was observed by ship radars and thermistor strings. Binary phase shift keying (BPSK) signals at the carrier frequencies of 813 and $1627 \mathrm{~Hz}$ are analyzed by time reversal processing followed by a single-channel DFE.

Two types of internal wave effects are investigated in the context of passive time reversal acoustic communications. One is the rapid channel fluctuation observed within 90-s data packets. It can be characterized as decreased channel coherence, which was the result of fast sound-speed perturbations during the internal wave passage. We show its effect on the time reversal receiver performance and apply channel tracking in the communication receiver to counteract such rapid fluctuation. The 
other one is the long-term (in the scale of hours) performance degradation in the depressed waveguide when the internal waves passed through the acoustic track. Significant acoustic intensity decrease was observed at the upper water column for several hours when the internal wave packet overlapped with the acoustic track. This is different from acoustic intensity fluctuations at low frequencies $(300-400 \mathrm{~Hz}$ or below) associated with the period of the internal waves, which are results of horizontal focusing and defocusing as reported in [6] and [7]. The index of refraction for horizontal rays has been shown to explain the horizontal focusing and defocusing at low frequencies [7]. At the considered frequencies ( 813 or $1627 \mathrm{~Hz}$ ), it is much smaller than at $300-400 \mathrm{~Hz}$ in the previous studies [6], [7]. This is because the index of refraction for horizontal rays is related to the modal amplitude, which is smaller at higher frequencies. Therefore, the horizontal focusing and defocusing are not considered as the primary reason for the observed intensity fluctuations. A simplified ray approximation is used to explain such acoustic intensity decrease and hence the acoustic communication performance degradation in the depressed waveguide.

The contribution of this paper is quantification of both shortterm and long-term acoustic fluctuation effects at the middle frequency band $(813$ and $1627 \mathrm{~Hz})$ in an internal wave experiment. These effects are measured by the communication performance through time reversal processing for an extended period (12 h). It should be noted that the carrier frequencies discussed here are relatively low. Acoustic communications at these frequencies can be used for long-range telemetry that requires only limited data rates. The observed internal wave effects on acoustic communications should apply to higher frequencies.

The paper is organized as follows. In Section II, the principle of passive time reversal acoustic communications will be briefly introduced. In Section III, the acoustic communication experiment during SW06 will be presented. In Section IV, sample acoustic data will be discussed and a time reversal receiver structure will be presented to analyze the communication data. In Section $\mathrm{V}$, the receiver performance during the experiment will be shown. Also, a simplified ray approximation will be put forward to explain the acoustic intensity decrease that causes communication performance degradation in the presence of the internal waves. Note that all time information in the paper is in coordinated universal time (UTC).

\section{Passive Time Reversal Acoustic Communications}

In a time reversal process, the source first transmits a probe that is recorded at the receiver. The received probe, serving as channel information, is time reversed and retransmitted at the receiver. Thus, sound focusing can be achieved at the source if the channel does not change significantly. This process can be implemented as passive time reversal or passive phase conjugation when a receiving array is available [17]. Here we consider a passive time reversal system in the ocean, which consists of a single transducer and an $M$-element hydrophone array. At the source, a binary information sequence $x(n)$ is transformed into a baseband continuous wave $x(t)$. Let $y^{(i)}(t)$ be the received baseband signal at the $i$ th hydrophone at the receiving array. The effect of the transmission medium between the source and $i$ th hydrophone can be characterized by a time-varying impulse response, $h^{(i)}(t, \tau), \quad 0<\tau<T_{L}$, where $T_{L}$ is the impulse response length. Therefore, $y^{(i)}(t)=x(t) * h^{(i)}(t, \tau)+v_{\mathrm{amb}}^{(i)}(t)$, where $v_{\mathrm{amb}}^{(i)}(t)$ represents the ambient noise and $*$ denotes the convolution operation. Note that as in [21], the received analog waveform is sampled at a fractional symbol interval to provide robustness to carrier phase fluctuations. However, the equations throughout the paper use symbol spaced signals for notational brevity.

In passive time reversal, the baseband signals on each channel $y^{(i)}(t)$ are matched-filtered by the time reversed channel estimates and the results are combined. The channel estimate is often obtained at the beginning of the data packet, denoted as $\hat{h}^{(i)}\left(t_{0}, \tau\right)$, by channel probes or known symbols. Therefore, the output of time reversal combining is

$$
r_{0}(t)=x(t) * q_{0}(t, \tau)+w_{0}(t)
$$

where $q_{0}(t, \tau)$ is the effective impulse response, or $q$-function [22], using $\hat{h}^{(i)}\left(t_{0}, \tau\right)$ in time reversal combining

$$
q_{0}(t, \tau)=\sum_{i=1}^{M}\left(\hat{h}^{(i)}\left(t_{0},-\tau\right)\right)^{*} * h^{(i)}(t, \tau)
$$

and ${ }^{*}$ denotes the complex conjugate and $w_{0}(t)$ is the noise component after time reversal combining.

Time reversal combining alone usually cannot achieve perfect equalization with a limited number of hydrophones. Therefore, a single-channel DFE with joint phase tracking [21] is followed to equalize the residual ISI in $r_{0}(t)$ of (1) [18], [19]. At low frequencies (below $3-5 \mathrm{kHz}$ ) with a static ocean condition, the impulse response $h^{(i)}(t, \tau)$ is usually considered constant during a data packet that has a duration of seconds to minutes. The single-channel DFE can compensate for small variations of the channel. An exponentially weighted recursive least squares (RLS) algorithm is often chosen to update the equalizer tap weights. The residual carrier phase offset in $r_{0}(t)$ is compensated for by a second-order phase locked loop embedded in the adaptive DFE. The output signal-to-noise ratio (SNR) is used to measure the performance of the receiver. It is the inverse of the mean squared error measured at the DFE soft output.

\section{The COMMUnication EXPERIMENT DuRING SW06}

SW06 was conducted on the New Jersey continental shelf from July to September 2006 [20]. In this paper, we focus on data collected between 18:00:00Z on August 17 and 06:00:00Z on August 18, when a packet of strong internal waves was observed by radars on the $R / V$ Sharp and the $R / V$ Oceanus as well as water temperature measurements [23]. The internal wave event was named Event 50 by the $R / V$ Sharp. The source was the Miami Sound Machine (MSM) [24]. During the experiment, it was moored at the depth of $70 \mathrm{~m}$ and at the range of 19.8 $\mathrm{km}$ from the 16-element Woods Hole Oceanographic Institution vertical line array (WHOI-VLA). The receiving array covered the depth from 13.5 to $77.75 \mathrm{~m}$. The water depth was about 80 $\mathrm{m}$ along the entire acoustic track. The experimental setting is shown in Fig. 1.

During Event 50, the $R / V$ Sharp and the $R / V$ Oceanus coordinated operations to monitor the origination and propagation of internal waves using onboard radars. At 18:00:00Z on August 


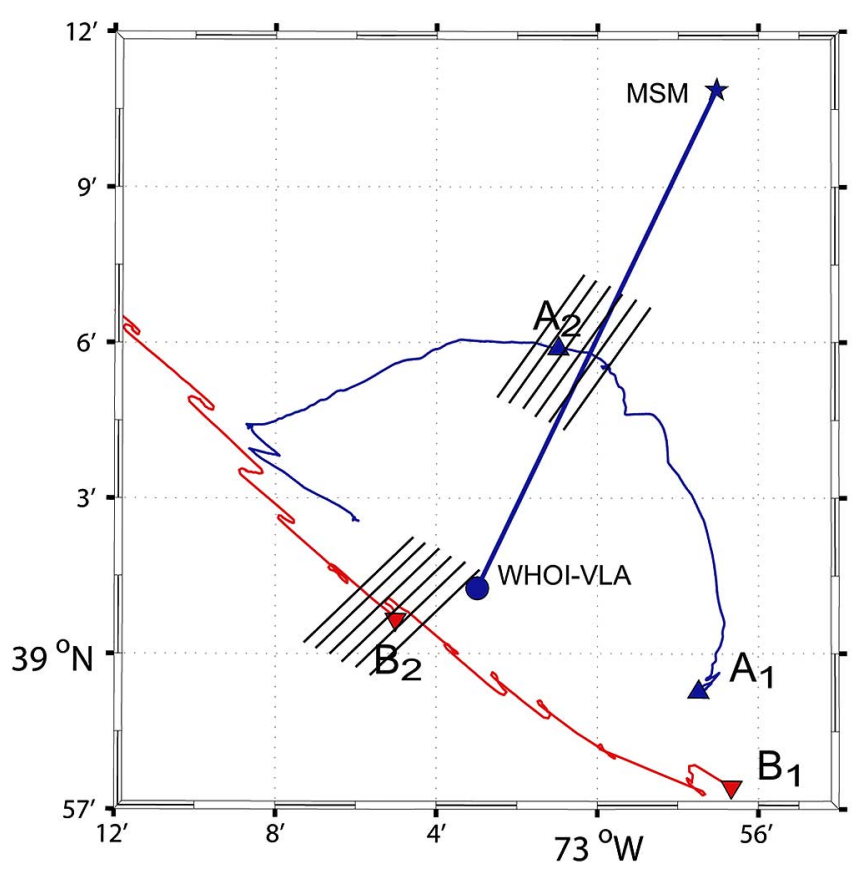

Fig. 1. Experimental setting. The MSM was the acoustic source and the WHOI-VLA was the receiver. The source-receiver range was about $19.8 \mathrm{~km}$. The water depth was about $80 \mathrm{~m}$. The ship tracks of the $R / V$ Sharp and the $R / V$ Oceanus were $A_{1}-A_{2}$ and $B_{1}-B_{2}$, respectively. The parallel lines around $A_{2}$ and $B_{2}$ illustrate the observed internal waves at 22:30:00Z on August 17, 2006.

17,2006 , the two vessels were stationary, with the $R / V$ Sharp at location $A_{1}$ and the $R / V$ Oceanus at location $B_{1}$ (Fig. 1), observing the origination of a packet of strong internal waves. As the packet propagated northwest, the two vessels followed the internal wave front. At around 21:15:00Z, the observed internal wave packet started to intersect the acoustic track at the receiving array. The observed internal wave packet is illustrated as the parallel lines in Fig. 1 at 22:30:00Z when the $R / V$ Sharp was at location $A_{2}$ and the $R / V$ Oceanus was at location $B_{2}$. The internal waves observed by the two vessels were known to be two segments of a singular nonlinear packet. As shown, the internal wave packet had a large overlap over the acoustic track at 22:30:00Z. The angle between the internal wave front and acoustic track was small, about $5^{\circ}$ observed from the $R / V$ Sharp around location $A_{2}$. The angle varied along the acoustic track because of the curvature of the internal wave front. From 00:00:00Z to 00:30:00Z, nearly the entire acoustic track was affected by the internal wave packet. At around 02:00:00Z on August 18 , the internal wave packet started to move off the acoustic track.

The internal wave packet was also observed by a network of thermistor strings deployed in the experimental site. Fig. 2 shows the temperature profiles recorded at the receiving array (WHOI mooring SW54) [20]. The SW54 mooring had 11 temperature sensors at 5-78.5 $\mathrm{m}$ that recorded the temperature profile every $30 \mathrm{~s}$. Before 21:15:00Z, there were no internal waves and the surface mixed layer extended to about $15-\mathrm{m}$ depth. Starting at 21:15:00Z, the water temperature exhibited fast perturbations when the internal waves passed through the receiving array. The surface mixed layer was depressed down to

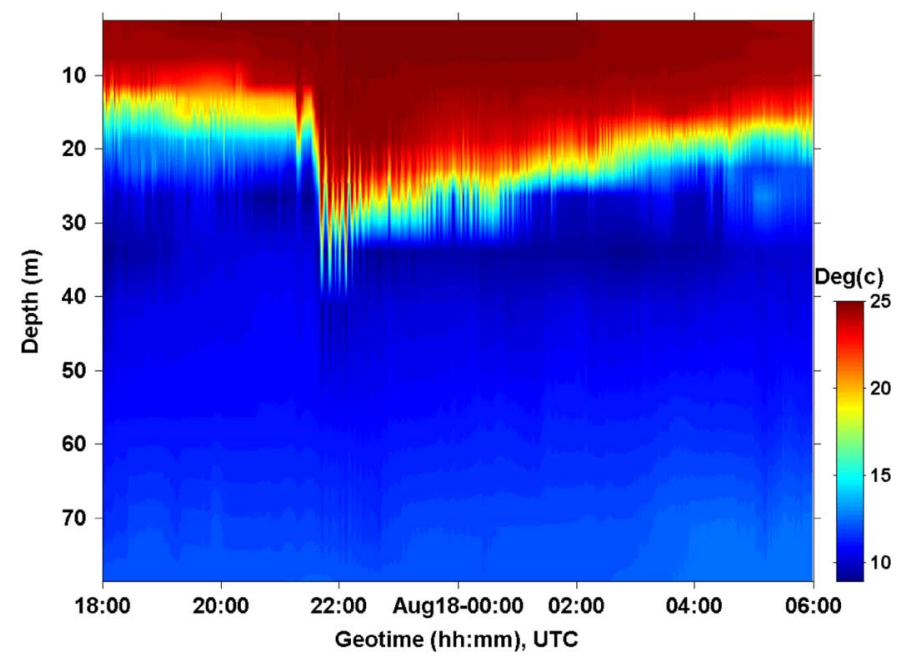

Fig. 2. Temperature profiles recorded at the receiving array mooring (WHOI mooring SW54) during Event 50.

a maximal depth of $35 \mathrm{~m}$ by the passing internal waves between 21:15:00Z and 02:00:00Z. After 02:00:00Z, the surface mixed layer slowly returned to its original thickness.

Two different pseudorandom BPSK signals, transmitted from the MSM in succession every 30 min during Event 50, will be analyzed here: 1) the 90-s BPSK signal at carrier frequency $f_{C}=813 \mathrm{~Hz}$ and 2) the 80-s BPSK signal at $f_{C}=1627 \mathrm{~Hz}$. The symbol rate $f_{R}$ of the signals is one fourth of the carrier frequency $f_{C}$, i.e., $f_{R}=f_{C} / 4$. The source level at the two carrier frequencies was about $186 \mathrm{~dB}$ re $1 \mu \mathrm{Pa}^{2} / \mathrm{Hz}$ at $1 \mathrm{~m}$.

\section{Passive Time Reversal Communications in the PRESENCE OF INTERNAL WAVES}

In this section, the rapid acoustic fluctuations caused by internal waves will be shown using sample data. Its impact on the performance of the time reversal receiver will be presented. Channel tracking will be applied to cope with such rapid acoustic fluctuations.

Due to the presence of the internal waves, the impulse response showed variable characteristics during Event 50. Fig. 3 shows the impulse responses at $813 \mathrm{~Hz}$ for two geotimes. They were obtained by the least squares (LS) estimation. As shown in Fig. 3(a), the impulse response was quite stable during the 90 $s$ at 18:00:00Z, before the internal waves reached the acoustic track. Such a stable arrival structure is beneficial to time reversal acoustic communications as will be shown later in this section. Let the channel correlation coefficient $\eta^{(i)}(\delta t)$ at the $i$ th channel be defined as

$$
\eta^{(i)}(\delta t)=\left|\frac{\left\langle\int_{\tau}\left(h^{(i)}(t, \tau)\right)^{*} h^{(i)}(t+\delta t, \tau)\right\rangle}{\sqrt{\left\langle\int_{\tau}\left|h^{(i)}(t, \tau)\right|^{2}\right\rangle\left\langle\int_{\tau}\left|h^{(i)}(t+\delta t, \tau)\right|^{2}\right\rangle}}\right|
$$

where $\langle\cdot\rangle$ denotes the ensemble average and $\delta t$ is the lag time. The calculated channel correlation based on the impulse responses in Fig. 3(a) was greater than 0.5 when $\delta t<65$ s. In other words, if channel coherence time is defined as the lag time at which $\eta^{(i)}(\delta t)=0.5$, then at 18:00:00Z it would be 65 s. In contrast, as shown in Fig. 3(b), the impulse response 


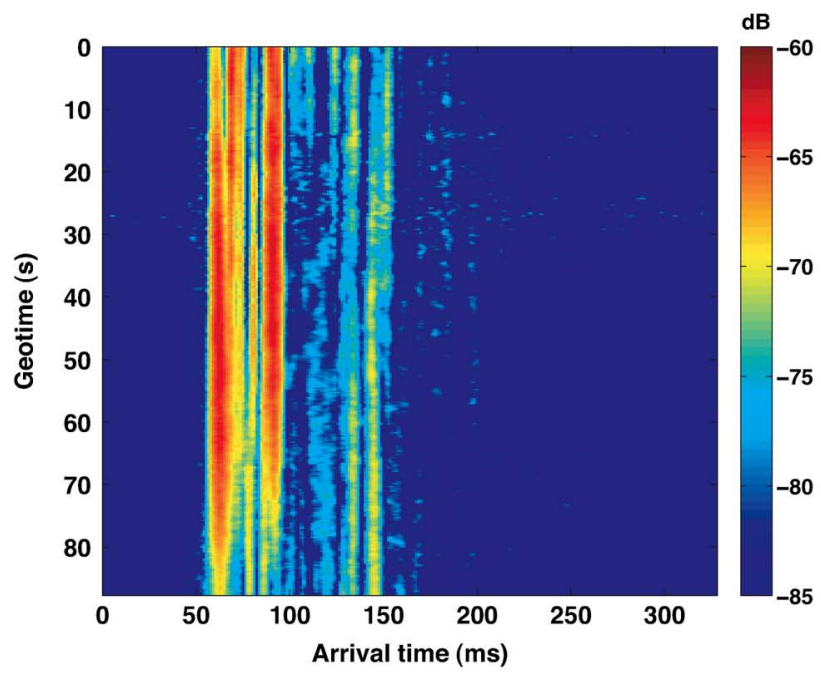

(a)

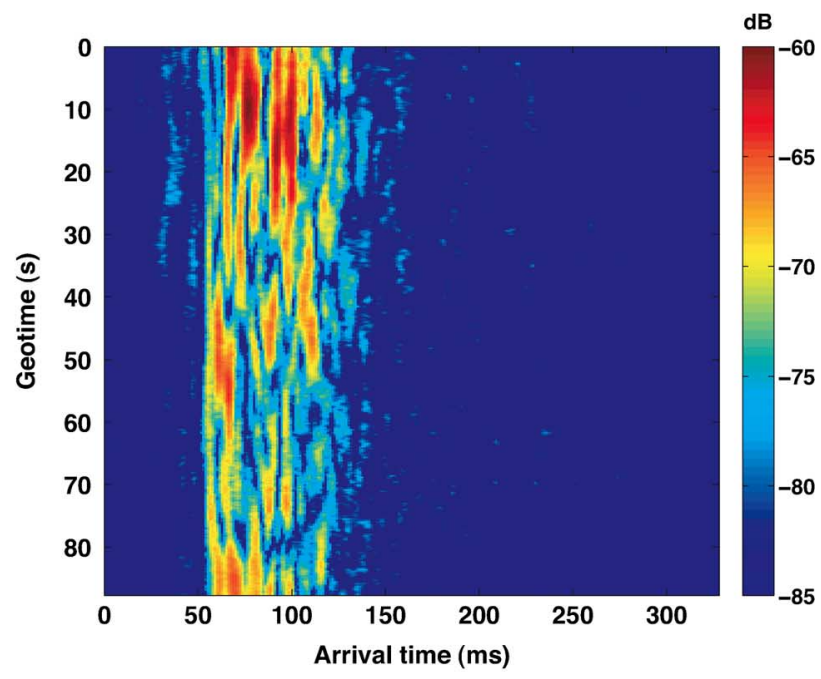

(b)

Fig. 3. Estimated impulse responses at $813 \mathrm{~Hz}$ (a) at 18:00:00Z and (b) at 22:30:00Z on August 17, 2006, respectively. The impulse responses were obtained for $69.75-\mathrm{m}$ depth at the receiving array.

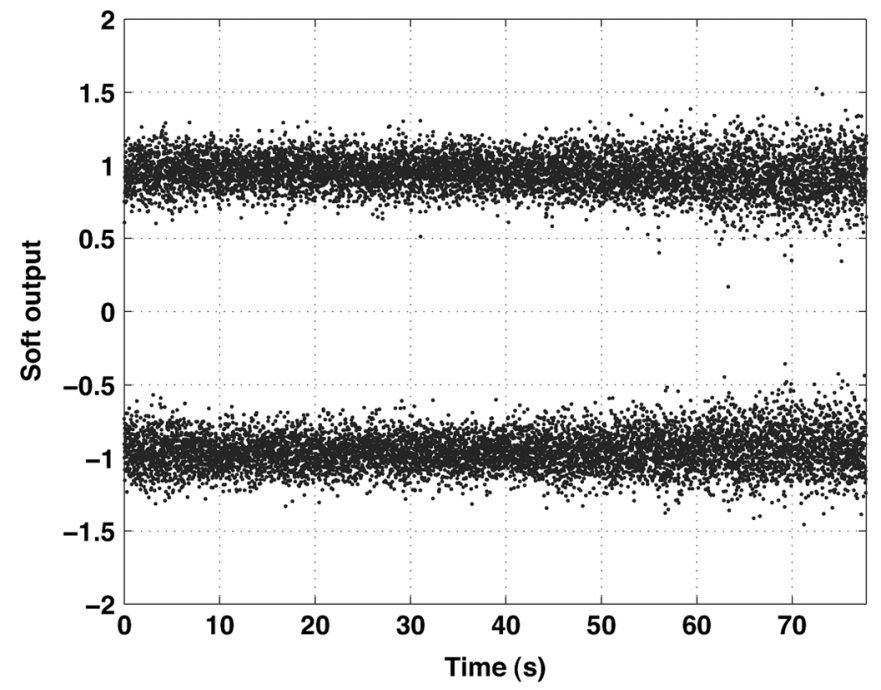

(a)

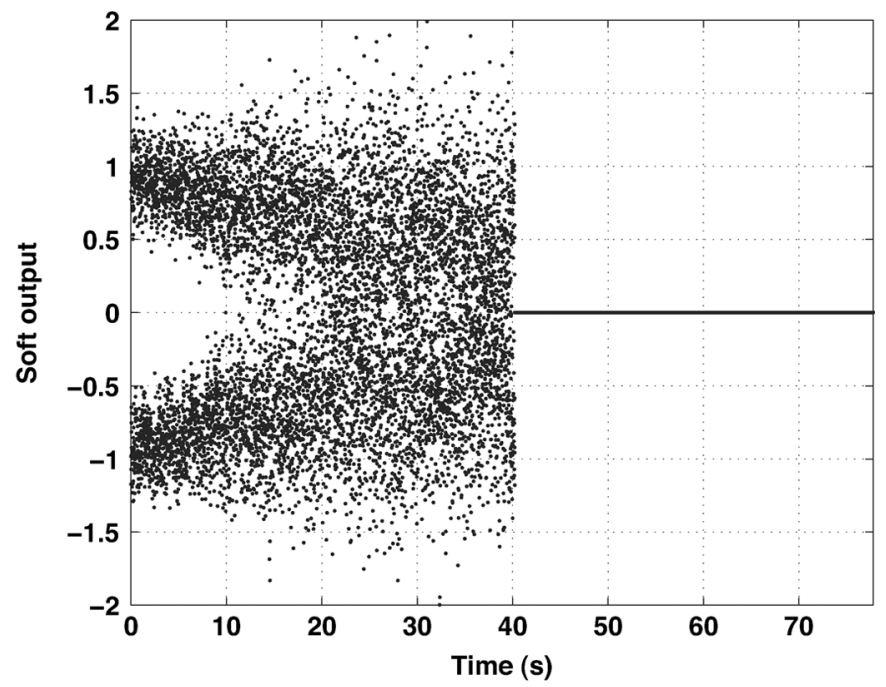

(b)

Fig. 4. Receiver soft output (a) at 18:00:00Z and (b) at 22:30:00Z on August 17, 2006 using the time reversal receiver without channel tracking.

fluctuated at a faster rate at 22:30:00Z when the internal waves passed through the acoustic track. The fast perturbations in the sound-speed profile caused this rapid channel fluctuation [11]. During this period, the channel coherence time was $15 \mathrm{~s}$. At $f_{C}=1627 \mathrm{~Hz}$, the impulse responses also demonstrated a sharp contrast between 18:00:00Z and 22:30:00Z (not shown in plots). The channel coherence times were $26 \mathrm{~s}$ at 18:00:00Z while $6 \mathrm{~s}$ at 22:30:00Z.

Such a difference in the channel characteristics can affect the performance of the time reversal receiver. As shown in Fig. 4(a), the time reversal receiver could successfully demodulate the data packet at 18:00:00Z with an output SNR of 14.6 dB. There were no demodulation errors for the 90-s data packet. But the receiver failed to demodulate the data packet at 22:30:00Z. As shown in Fig. 4(b), the receiver had significant demodulation errors from $20 \mathrm{~s}$ onwards. Around $40 \mathrm{~s}$, the DFE diverged and the receiver failed. This was because the channel varied sig- nificantly within the data packet. When the channel fluctuated, pulse compression could not be achieved through time reversal combining in (1) and (2) because of the nonnegligible mismatch between $\hat{h}^{(i)}\left(t_{0}, \tau\right)$ and $h^{(i)}(t, \tau)$.

To deal with the channel fluctuation in the presence of internal waves, we propose to update the channel estimates regularly within a data packet at time intervals of $T_{U}$. Note that time reversal combining alone with frequent channel updates has been discussed for high frequencies [25]. Joint time reversal combining and multichannel equalization have been considered for moving platforms [26]. In [26], only one combining coefficient per hydrophone channel is updated when the impulse responses fluctuate. Both channel tracking and phase tracking have also been incorporated into time reversal receivers at high frequencies $(12 \mathrm{kHz})$ in dynamic ocean environments [4]. Channel estimation can be performed based on the past demodulation results using the LS algorithm. Once the updated channel esti- 


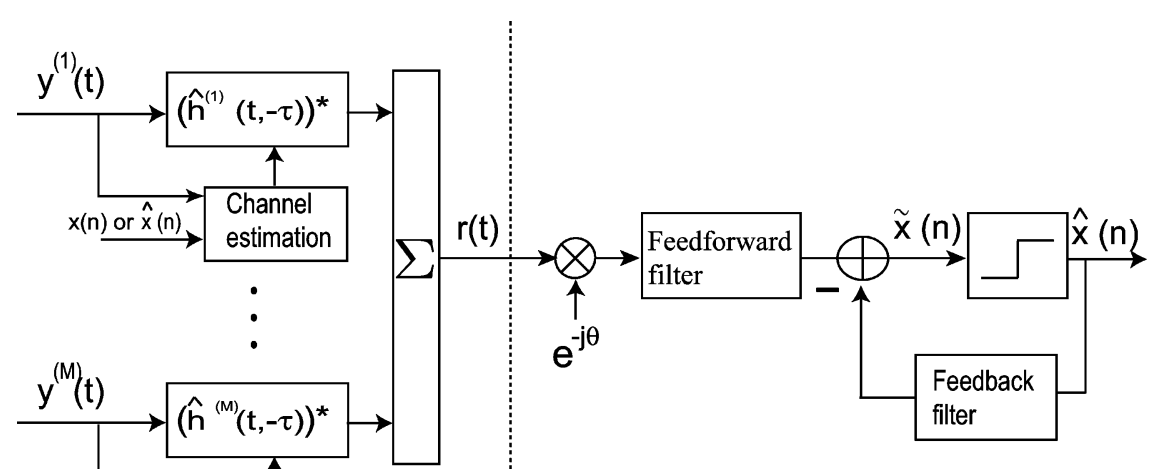

Single channel DFE

Channel estimation and

time reversal multichannel combining

Fig. 5. Time reversal receiver with channel tracking.

mates $\hat{h}^{(i)}(t, \tau)$ are available, they are used in time reversal combining. Fig. 5(a) shows the receiver structure. So the $q$-function becomes

$$
q(t, \tau)=\sum_{i=1}^{M}\left(\hat{h}^{(i)}(t,-\tau)\right)^{*} * h^{(i)}(t, \tau) .
$$

With frequent updates to track the channel, the $q$-function $q(t, \tau)$ can be almost constant or slowly varying even for fluctuating impulse responses.

At the beginning of a data packet, a preamble (or a sequence of known symbols) is used to perform initial channel estimation and to adaptively train the DFE tap weights. After the preamble, the channel estimates are updated at time intervals of $T_{U}$. The channel estimate $\hat{h}^{(i)}(t, \tau)$ can be obtained from the received signal and the previously detected symbols $\hat{x}(n)$. With the updated channel estimate $\hat{h}^{(i)}(t, \tau)$, time reversal combining is performed and the DFE generates the next block of demodulation results. The demodulation process continues until it reaches the end of the data packet.

\section{A. The Channel Update Interval $T_{u}$}

The choice of the channel update interval $T_{U}$ is dependent on the fluctuating rate of the channel. Generally, a more dynamic channel requires more frequent channel updates. Smaller channel update intervals $T_{U}$, i.e., more frequent channel updates, lead to better receiver performance for fluctuating channels. At 18:00:00Z, the output SNR decreased gradually with the increase of $T_{U}$ at $f_{C}=813 \mathrm{~Hz}$. Even without channel updates in the data packets, the time reversal receiver was able to demodulate all the symbols without any errors. But at 22:30:00Z, the output SNR decreased much faster with the increase of $T_{U}$. The receiver failed, the bit error rate (BER) approaching 0.5 , at $T_{U}=10 \mathrm{~s}$. At both 18:00:00Z and 22:30:00Z, additional decreasing of $T_{U}$ below $1 \mathrm{~s}$ resulted in minimal performance improvement, with or without internal waves present. At $f_{C}=1627 \mathrm{~Hz}$, similar results can be observed.

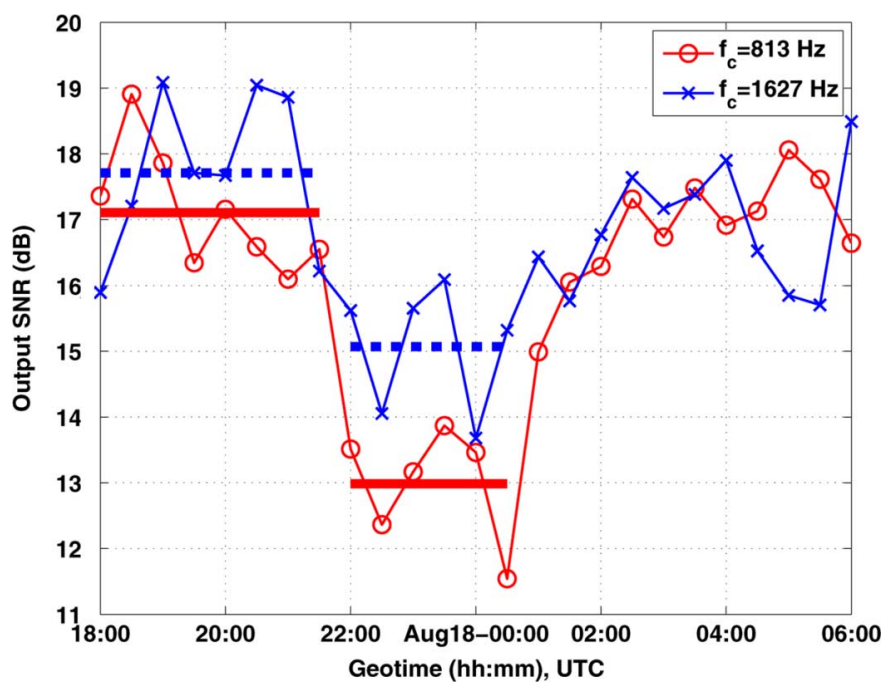

Fig. 6. Output SNR of the receiver at both carrier frequencies during Event 50. The horizontal lines and dashed lines indicate the average output SNRs before and during the internal wave passage at 813 and $1627 \mathrm{~Hz}$, respectively.

\section{COMmunication Performance During SW06}

In this section, the performance of the time reversal receiver with channel tracking is shown for the selected 12-h period in SW06. A simplified ray approximation is also presented to explain the acoustic intensity fluctuations that caused long-term communication performance degradation in the presence of internal waves.

In the time reversal receiver with channel tracking, 14 elements of the receiving array that covered the water column at depths of 13.5-77.25 m were used. The received data were oversampled with a rate of $K=3$. At the beginning of the data packet, a $T_{P}=5 \mathrm{~s}$ preamble was used to carry out initial channel estimation and DFE tap weight training. The estimated impulse response length was $T_{L}=330 \mathrm{~ms}$ at $f_{c}=813 \mathrm{~Hz}$ and $T_{L}=250 \mathrm{~ms}$ at $f_{C}=1627 \mathrm{~Hz}$. The observation time span used in the LS channel estimation is denoted as $T_{E}$, which was set as twice the estimated impulse response length. The channel 


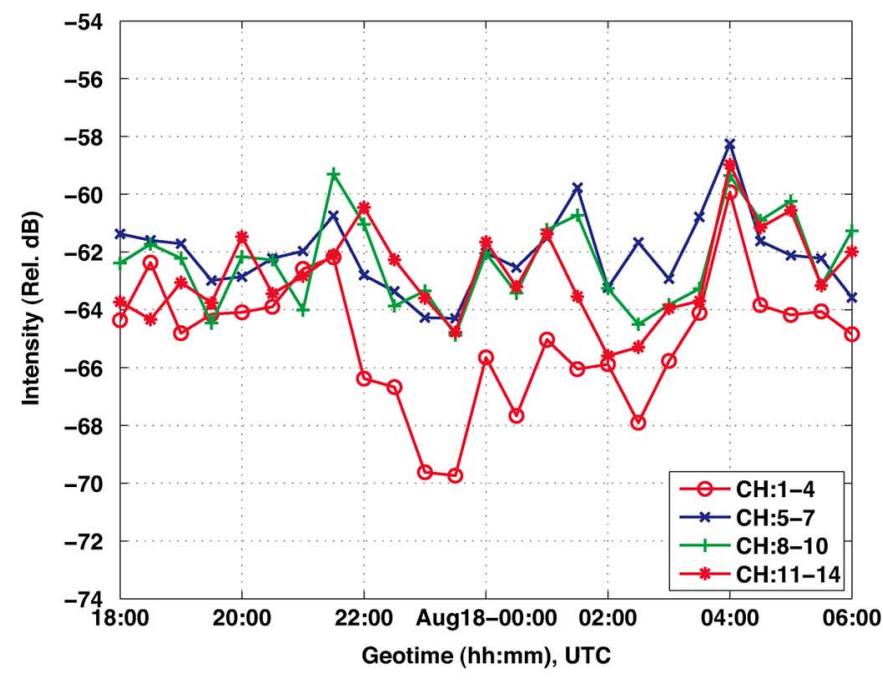

(a)

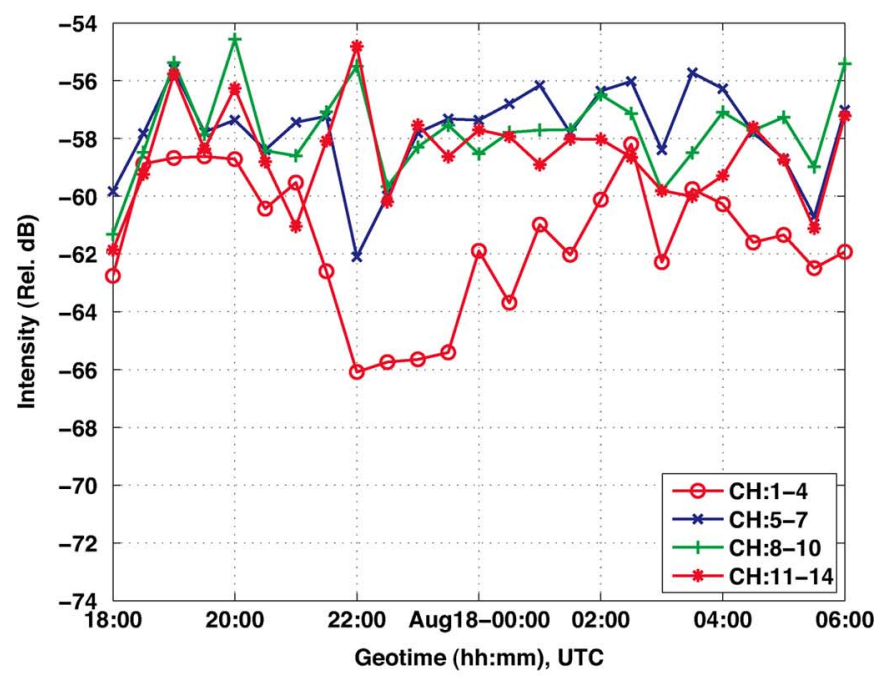

(b)

Fig. 7. Average intensity (a) at $813 \mathrm{~Hz}$ and (b) at $1627 \mathrm{~Hz}$ at four groups of hydrophones.

update interval was also set as twice the estimated impulse response length. Therefore, $T_{U}=T_{E}=2 T_{L}$. The number of the feedforward taps was $K N_{\mathrm{ff}}=30$ for the fractionally spaced DFE where $N_{\mathrm{ff}}=10$ was the feedforward filter span in symbols. The number of the feedback taps was $N_{\mathrm{fb}}=5$. The RLS forgetting factor in the DFE was 0.998 .

Fig. 6 shows the output SNR at the two carrier frequencies during Event 50. As shown, the output SNR at both carrier frequencies decreased during the period from 22:00:00Z to 00:30:00Z when the internal waves passed through the acoustic track. As shown in Fig. 2, the water column had an extended surface mixed layer and strong temperature fluctuations during this period. The average output SNRs before and during the passage of the internal waves are marked in Fig. 6 for both carrier frequencies. At $f_{C}=813 \mathrm{~Hz}$, the performance degradation caused by the internal waves was significant: The average difference in the output SNR was $4 \mathrm{~dB}$ between the condition when this internal wave packet did not reach the acoustic track and the condition when internal waves passed through the acoustic track. At $f_{C}=1627 \mathrm{~Hz}$, the average difference in the output SNR was about $3 \mathrm{~dB}$. The minimum output SNR occurred at 00:00:00Z (for $f_{C}=813 \mathrm{~Hz}$ ) and 00:30:00Z (for $f_{C}=1627 \mathrm{~Hz}$ ) on August 18, 2006, when the entire acoustic track was affected by the passing internal waves.

During the 12-h period, most of the data packets had no demodulation errors. Only one out of 25 packets had demodulation errors at the carrier frequency of $813 \mathrm{~Hz}$ and the BER was below $10^{-4}$. Three packets at the carrier frequency of $1627 \mathrm{~Hz}$ had demodulation errors, with BERs also below $10^{-4}$. The average output SNR during the 12-h period was slightly higher at 1627 Hz: it was $16.0 \mathrm{~dB}$ for $f_{C}=813 \mathrm{~Hz}$ and $16.7 \mathrm{~dB}$ for $f_{C}=1627$ Hz. Generally, the communication performance at higher carrier frequencies is worse than that at lower carrier frequencies. Note that the double bandwidth was used for double symbol rate transmission at $f_{C}=1627 \mathrm{~Hz}$. However, the average received signal intensity was $4.2 \mathrm{~dB}$ higher at $1627 \mathrm{~Hz}$ as will be shown in Fig. 7. The average was performed over the receiving array for the 12-h period. The higher signal intensity at $1627 \mathrm{~Hz}$ was attributed to the combination of similar source spectral levels at the two carrier frequencies and double bandwidth transmission at $1627 \mathrm{~Hz}$. Considering the half symbol period at $f_{C}=1627$ $\mathrm{Hz}$, the symbol energy was still $1.2 \mathrm{~dB}$ higher at $1627 \mathrm{~Hz}$. In the experiment, the noise spectral density decreased as the frequency increased. The average noise spectral density was 3.8 $\mathrm{dB}$ lower at the $1627-\mathrm{Hz}$ band. This resulted in an average of 5-dB higher input SNR per symbol and a slightly better communication performance at $f_{C}=1627 \mathrm{~Hz}$.

\section{A. Ray Approximation in the Presence of Internal Waves}

Fig. 7 shows the received intensity at the receiving array for two carrier frequencies during the 12-h period. Note that the intensity numbers at 813 and $1627 \mathrm{~Hz}$ were normalized by the same factor. The 14 elements have been divided into four groups and the intensity was averaged over the three or four hydrophones in the groups. At the top four hydrophones (denoted as $\mathrm{CH}:$ 1-4) average acoustic intensity decreased about 6-8 $\mathrm{dB}$ at both carrier frequencies during the period when the internal waves overlapped with the acoustic track. Acoustic intensity in the middle or lower water column did not show remarkable difference between before and during the internal waves passage. The intensity decrease led to the lower output SNR in the presence of internal waves since the received intensity was directly related to input SNR and, therefore, the communication performance [27].

In [6] and [7], significant acoustic intensity fluctuations at low frequencies (300-400 $\mathrm{Hz}$ or below) have been reported at the internal wave periods (10-15 min in SWARM-95 or SW06 environments). Horizontal focusing and defocusing have been considered to explain the acoustic intensity fluctuations. The frequency-dependent index of refraction for horizontal rays is quite remarkable at 300-400 Hz. However, at the considered frequencies ( 813 or $1627 \mathrm{~Hz}$ ), it is much smaller [8]. Therefore, the horizontal focusing and defocusing should not have significant contributions towards the observed intensity fluctuations at a much larger time scale. In order to explain the observed intensity fluctuations, a simplified ray approximation is proposed in this section. 


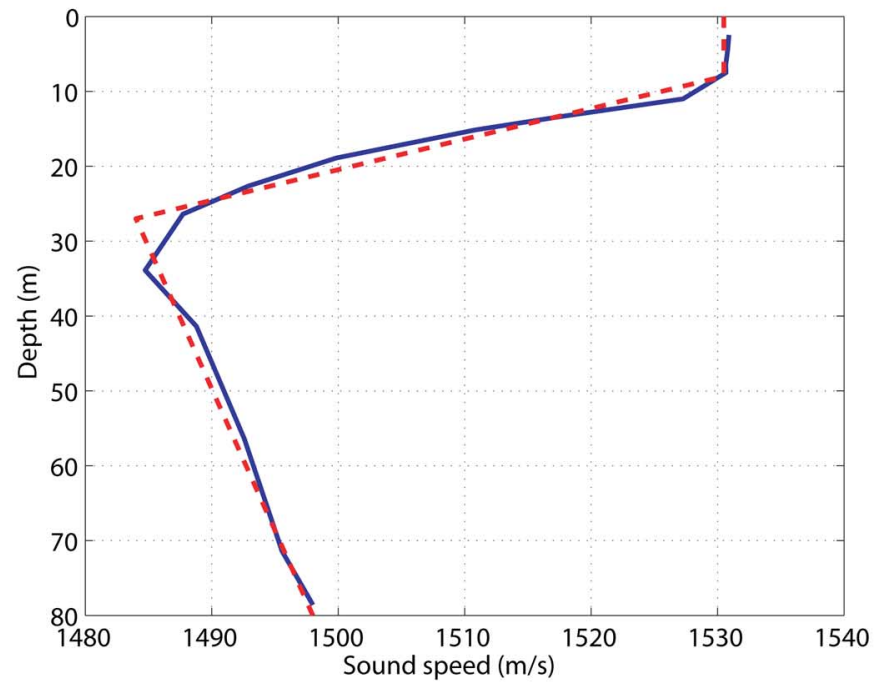

(a) 18:00 on Aug. 17 (no internal waves)

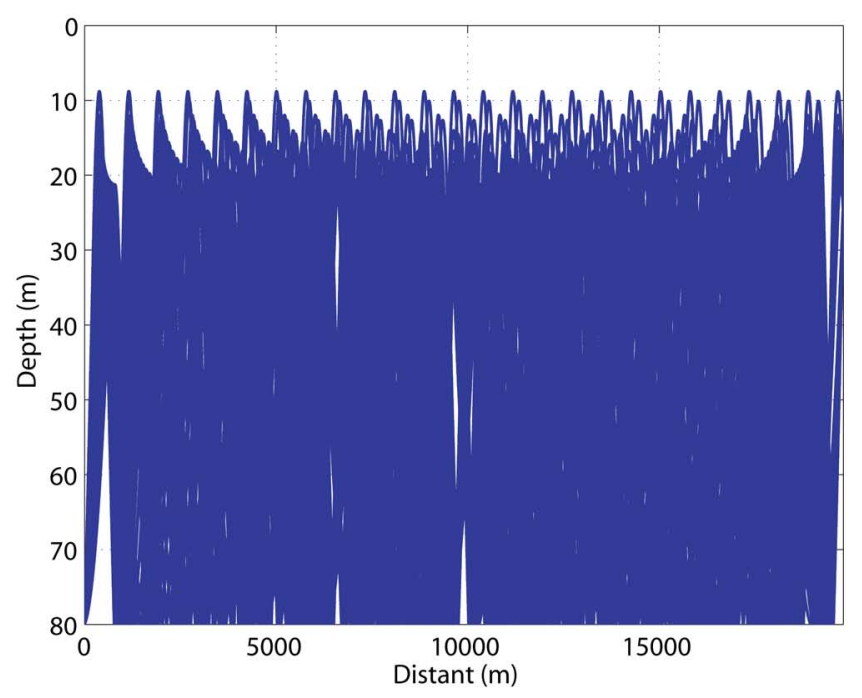

(c) Ray diagram at 18:00 (no internal waves)

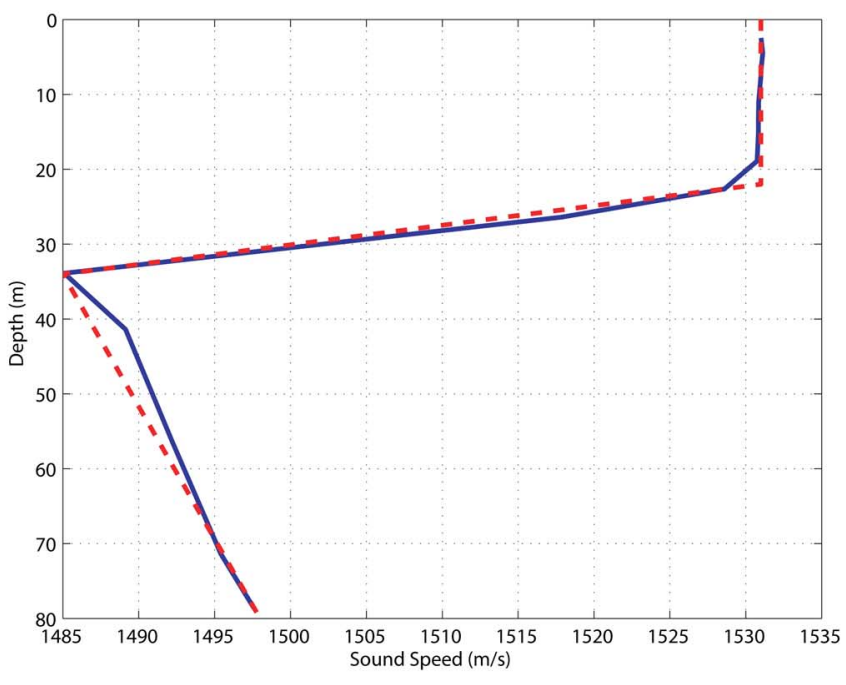

(b) 22:30 on Aug. 17 (with internal waves)

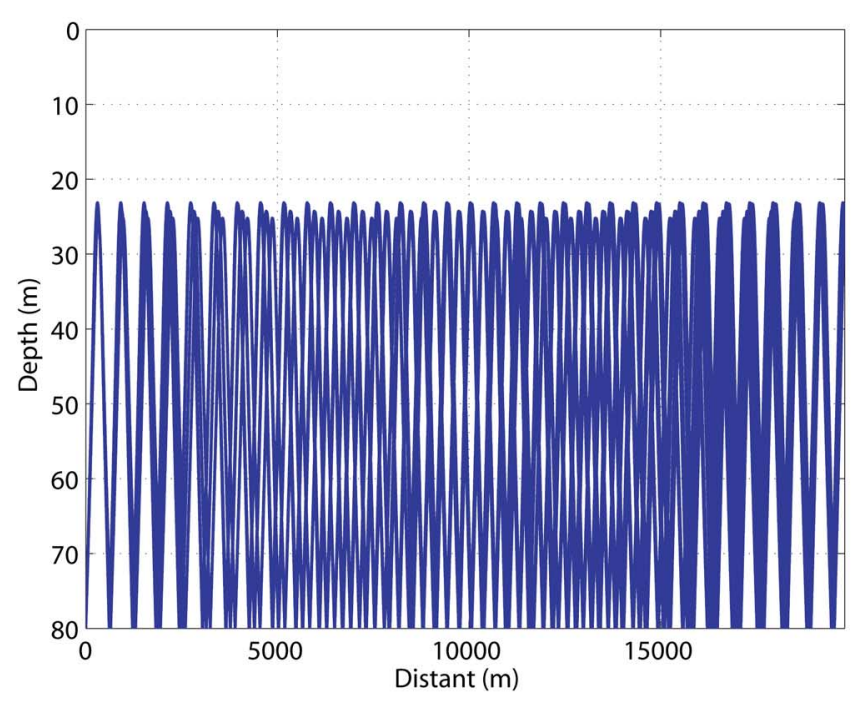

(d) Ray diagram at 22:30 (with internal waves)

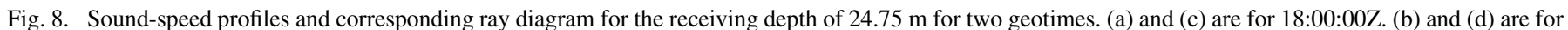
22:30:00Z. The dashed lines in (a) and (b) indicate the simplified profiles used in the ray tracing.

As shown in Fig. 2, lowering of the thermocline layer was observed when the internal waves passed through the acoustic track from 21:15:00Z to 02:00:00Z. Fig. 8(a) and (b) shows the sound-speed profiles of the water column without (18:00:00Z) and with (22:30:00Z) the presence of internal waves, respectively. In both geotimes, the sound-speed profiles were averaged over a 30-min period. At 22:30:00Z, the waveguide was depressed along the acoustic track and the extent of depression varied at different parts. The average over 30 min gives an estimate of the waveguide depression. As shown in Fig. 8(a) and (b), the thermocline was about $10 \mathrm{~m}$ lower at 22:30:00Z (internal wave present) than it was at 18:00:00Z (before the internal wave arrival). When the internal waves passed through the acoustic track, the water column experienced fast perturbations, in addition to the lowering of the thermocline. As mentioned in Section IV, the fast perturbations in the sound-speed profile caused rapid channel fluctuation, which can be addressed by the channel tracking in the time reversal processor. Here we only consider the effect of the depressed waveguide.

As shown in Fig. 8(a) and (b), sound-speed profiles in the waveguide had steep negative gradients in the thermocline layer. Therefore, the main contributions to the sound intensity were the rays refracted in the thermocline for this waveguide. In the ray approximation presented in this section, only the rays refracted in the thermocline layer are considered. We also assume that the sound-speed profiles and bottom properties are range independent in the approximation.

At 22:30:00Z, no rays refracted in the thermocline layer could reach the top three hydrophones. In other words, the top three hydrophones were in the shadow zone because of the depressed waveguide. Two additional hydrophones, at 24.75 
TABLE I

Modeled Intensity Decrease at the Receiving ARray Through Ray Approximation ( $S$ Denotes That the Hydrophone Was Shadowed; A Negative Decibel Number IndiCATES INCREASEd INTENSITY With THE PRESENCE OF INTERNAL WaVeS)

\begin{tabular}{|l|l|l|l|l|l|l|l|}
\hline \hline Depth & $13.5 \mathrm{~m}$ & $17.25 \mathrm{~m}$ & $21.0 \mathrm{~m}$ & $24.75 \mathrm{~m}$ & $28.5 \mathrm{~m}$ & $32.25 \mathrm{~m}$ & $36.0 \mathrm{~m}$ \\
\hline Intensity decrease & $S$ & $S$ & $S$ & $9.3 \mathrm{~dB}$ & $2.1 \mathrm{~dB}$ & $1.9 \mathrm{~dB}$ & $0.3 \mathrm{~dB}$ \\
\hline \hline Depth & $39.75 \mathrm{~m}$ & $43.5 \mathrm{~m}$ & $47.25 \mathrm{~m}$ & $54.75 \mathrm{~m}$ & $62.25 \mathrm{~m}$ & $69.75 \mathrm{~m}$ & $77.25 \mathrm{~m}$ \\
\hline Intensity decrease & $-0.6 \mathrm{~dB}$ & $0.1 \mathrm{~dB}$ & $-0.3 \mathrm{~dB}$ & $-0.2 \mathrm{~dB}$ & $-0.4 \mathrm{~dB}$ & $0.1 \mathrm{~dB}$ & $-0.1 \mathrm{~dB}$ \\
\hline
\end{tabular}

and $28.5 \mathrm{~m}$, received less acoustic intensity, though they were not in the shadow zone according to the ray approximation. Fig. 8(c) and (d) shows the ray tracing results for the receiving depth of $24.75 \mathrm{~m}$ without and with the presence of internal waves, respectively. Only eigenrays refracted in the thermocline are plotted. As shown in Fig. 8(c) and (d), when the thermocline layer was in a lower position, fewer rays could reach the receiving hydrophone. Further, these rays had smaller ray cycles and larger grazing angles.

On the basis of the measured sound-speed profiles and bottom parameters, the acoustic intensity decrease caused by the depressed waveguide can be estimated. The transmission loss of the $l$ th ray is determined by the attenuation coefficient $\gamma_{l}(\chi)$, which in turn is determined by its ray cycle and reflection coefficient [28], [29]

$$
\gamma_{l}(\chi)=\frac{\ln \left|V_{l}(\chi)\right|}{D_{l}(\chi)}
$$

where $D_{l}(\chi)$ is the ray cycle, $V_{l}(\chi)$ is the Fresnel reflection coefficient, and $\chi$ is the grazing angle of the ray. Both $V_{l}(\chi)$ and $D_{l}(\chi)$ are dependent on the grazing angle $\chi$ and on waveguide parameters such as sound-speed profiles and bottom properties. The Fresnel reflection coefficient $V_{l}(\chi)$ is defined as

$$
V_{l}(\chi)=\frac{m_{1} \sin \chi-\sqrt{n_{1}^{2}(1+i \alpha)-\cos ^{2} \chi}}{m_{1} \sin \chi+\sqrt{n_{1}^{2}(1+i \alpha)-\cos ^{2} \chi}}
$$

where $m_{1}, n_{1}$, and $\alpha$ are the density ratio, the refractive index, and the absorptive coefficient of the bottom, respectively. The sound speed of the bottom was chosen as $1610 \mathrm{~m} / \mathrm{s}$, the density as $1800 \mathrm{~kg} / \mathrm{m}^{3}$, and the absorptive coefficient as 0.01 . These parameters were measured during SWARM-95 [30] and SW06 [31]. The amplitude of the $l$ th ray is

$$
A_{l} \sim \exp \left(\gamma_{l}(\chi) R\right)
$$

where $R$ is the source-receiver range. Note that $\left|V_{l}(\chi)\right|<1$ here and it decreases with the increase of the grazing angle. According to (6) and (7), either larger grazing angles or smaller ray cycles will result in smaller ray amplitude. Therefore, rays with smaller cycles and larger grazing angles at 23:00:00Z had smaller amplitudes than those at 18:00:00Z. At the receiving depth of $24.75 \mathrm{~m}$, the intensity at 22:30:00Z (in the presence of internal waves) was $9.3 \mathrm{~dB}$ less than at 18:00:00Z (without the presence of internal waves). Also as mentioned, the top three hydrophones were in the shadow zone because of the depressed waveguide. Therefore, the average intensity decrease as shown in Fig. 7 was significant at the top hydrophones. At this and other depths, the calculated ray cycle was about $600 \mathrm{~m}$ for the depressed waveguide and $800 \mathrm{~m}$ for the not depressed case. Another separate effort on SW06 showed similar results for the ray cycle calculation [32]. The average number of bottom bounces was about 20-30 times for the 19.8-km acoustic track.

Table I shows the intensity decrease calculation via the ray approximation at the receiving array. In addition to the modeled intensity decrease at top hydrophones, the modeled intensity at the middle and lower water column was shown not affected significantly. When the thermocline was depressed, the total acoustic energy would remain unchanged compared to the not depressed waveguide only if there was no bottom attenuation. The hydrophones at the middle and lower water column would receive higher intensity. However, with bottom attenuation, the depressed thermocline generated eigenrays with smaller ray cycles and these eigenrays attenuated more because of more bottom bounces. This led to minimum or no acoustic intensity increase at the middle or lower water column. The total intensity across the water column decreased.

\section{CONCLUSION}

During a 12-h period in SW06, detailed environmental measurements were made to study internal wave effects on coherent underwater acoustic communications. BPSK signals were transmitted while a strong packet of internal waves passed through the entire 19.8-km acoustic track. The communication data were analyzed using passive time reversal processing followed by a single-channel DFE. It was shown that in the presence of internal waves the time reversal receiver had to track channel variations due to disturbed channel coherence. We demonstrated that successful data communications had been achieved for the 12-h period when the internal waves passed through. However, even with channel tracking, the time reversal receiver experienced significant performance degradation, average 3-4-dB decrease in the output SNR, due to the acoustic intensity decrease caused by the passing internal waves. A simplified ray approximation was proposed to explain the acoustic intensity decrease that caused communication performance degradation in the presence of the internal waves.

\section{ACKNOWLEDGMENT}

The authors would like to thank all participants of SW06, especially the science crew aboard the $R / V$ Sharp: L. Brown, S. Crocker, G. Dossot, J. Largeaud, J. Luo, J. Siegel, L. Wan, and J. Yang. They would also like to thank the $R / V$ Sharp crew for their help during the experiment.

\section{REFERENCES}

[1] D. B. Kilfoyle and A. B. Baggeroer, "The state of the art in underwater acoustic telemetry," IEEE J. Ocean. Eng., vol. 25, no. 1, pp. 4-27, Jan. 2000.

[2] N. M. Carbone and W. S. Hodgkiss, "Effects of tidally driven temperature fluctuations on shallow-water acoustic communications at 18 kHz," IEEE J. Ocean. Eng., vol. 25, no. 1, pp. 84-94, Jan. 2000. 
[3] J. C. Preisig, "Performance analysis of adaptive equalization for coherent acoustic communications in the time-varying ocean environment," J. Acoust. Soc. Amer., vol. 118, no. 1, pp. 263-278, Jul. 2005.

[4] A. Song, M. Badiey, H.-C. Song, W. S. Hodgkiss, M. B. Porter, and the KauaiEx Group, "Impact of ocean variability on coherent underwater acoustic communications during the Kauai experiment (KauaiEx)," J. Acoust. Soc. Amer., vol. 123, no. 2, pp. 856-865, Feb. 2008.

[5] J. R. Apel, M. Badiey, C.-S. Chiu, S. Finette, R. Headrick, J. Kemp, J. F. Lynch, A. Newhall, M. H. Orr, B. H. Pasewark, D. Tielbuerger, A. Turgut, K. von der Heydt, and S. Wolf, "An overview of the 1995 SWARM shallow-water internal wave acoustic scattering experiment," IEEE J. Ocean. Eng., vol. 22, no. 3, pp. 465-500, Jul. 1997.

[6] M. Badiey, Y. Mu, J. Lynch, J. R. Apel, and S. Wolf, "Temporal and azimuthal dependence of sound propagation in shallow water with internal waves," IEEE J. Ocean. Eng., vol. 27, no. 1, pp. 117-129, Jan. 2002.

[7] M. Badiey, B. G. Katsnelson, J. F. Lynch, S. Pereselkov, and W. L. Siegmann, "Measurement and modeling of three-dimensional sound intensity variations due to shallow-water internal waves," J. Acoust. Soc. Amer., vol. 117, no. 2, pp. 613-625, Feb. 2005.

[8] M. Badiey, B. G. Katsnelson, J. F. Lynch, and S. Pereselkov, "Frequency dependence and intensity fluctuations due to shallow water internal waves," J. Acoust. Soc. Amer., vol. 122, no. 2, pp. 747-760, Aug. 2007.

[9] R. H. Headrick, J. F. Lynch, J. N. Kemp, A. E. Newhall, K. von der Heydt, J. Apel, M. Badiey, C.-S. Chiu, S. Finette, M. Orr, B. Pasewark, A. Turgot, S. Wolf, and D. Tielbuerger, "Acoustic normal mode fluctuation statistics in the 1995 SWARM internal wave scattering experiment," J. Acoust. Soc. Amer., vol. 107, no. 1, pp. 201-220, Jan. 2000.

[10] D. Rouseff, A. Turgut, S. N. Wolf, S. Finette, M. H. Orr, B. H. Pasewark, J. R. Apel, M. Badiey, C.-S. Chiu, R. H. Headrick, J. F. Lynch, J. N. Kemp, A. E. Newhall, K. von der Heydt, and D. Tielbuerger, "Coherence of acoustic modes propagating through shallow water internal waves," J. Acoust. Soc. Amer., vol. 111, no. 4, pp. 1655-1666, Apr. 2002.

[11] K. B. Yoo, "Shallow water temporal coherence of broadband acoustic signals in the presence of internal waves," IEEE J. Ocean. Eng., vol. 30, no. 2, pp. 391-405, Apr. 2005.

[12] T. C. Yang, "Measurements of temporal coherence of sound transmissions through shallow water," J. Acoust. Soc. Amer., vol. 120, no. 5, pp. 2595-2614, Oct. 2006.

[13] T. C. Yang, "The effect of internal waves on low frequency underwater acoustic communications," in Proc 18th Int. Congr. Acoust., Kyoto, Japan, Apr. 2004, pp. 975-978.

[14] A. Parvulescu and C. S. Clay, "Reproducibility of signal transmissions in the ocean," Radio Electr. Eng., vol. 29, pp. 223-228, 1965.

[15] W. A. Kuperman, W. S. Hodgkiss, H. C. Song, T. Akal, C. Ferla, and D. R. Jackson, "Phase conjugation in the ocean: Experimental demonstration of an acoustic time-reversal mirror," J. Acoust. Soc. Amer., vol. 103, no. 1, pp. 25-40, Jan. 1998.

[16] G. F. Edelmann, T. Akal, W. S. Hodgkiss, S. Kim, W. A. Kuperman, and H. C. Song, "An initial demonstration of underwater acoustic communications using time reversal," IEEE J. Ocean. Eng., vol. 31, no. 3, pp. 602-609, Jul. 2002.

[17] D. Rouseff, D. R. Jackson, W. L. J. Fox, C. D. Jones, J. A. Ritcey, and D. R. Dowling, "Underwater acoustic communication by passive-phase conjugation: Theory and experimental results," IEEE J. Ocean. Eng., vol. 26, no. 4, pp. 821-831, Oct. 2001.

[18] T. C. Yang, "Correlation-based decision-feedback equalizer for underwater acoustic communications," IEEE J. Ocean. Eng., vol. 30, no. 4, pp. 865-880, Oct. 2005.

[19] H. C. Song, W. S. Hodgkiss, W. A. Kuperman, M. Stevenson, and T. Akal, "Improvement of time reversal communications using adaptive channel equalizers," IEEE J. Ocean. Eng., vol. 31, no. 2, pp. 487-496, Apr. 2006.

[20] A. E. Newhall, T. F. Duda, J. D. Irish, K. von der Heydt, J. N. Kemp, S. A. Lerner, S. P. Liberatore, Y.-T. Lin, J. F. Lynch, A. R. Maffei, A. K. Morozov, A. Shmelev, C. J. Sellers, and W. E. Witzell, "Acoustic and oceanographic observations and configuration information for the WHOI moorings for the SW06 experiment," Woods Hole Ocean. Inst. (WHOI), Woods Hole, MA, Tech. Rep. WHOI-2007-04, 2007.

[21] M. Stojanovic, J. A. Catipovic, and J. G. Proakis, "Phase-coherent digital communications for underwater acoustic channels," IEEE J. Ocean. Eng., vol. 19, no. 1, pp. 100-111, Jan. 1994.
[22] T. C. Yang, "Temporal resolutions of time-reversal and passive-phase conjugation for underwater acoustic communications," IEEE J. Ocean. Eng., vol. 28, no. 2, pp. 229-245, Apr. 2003.

[23] J. Luo, M. Badiey, E. A. Karjadi, B. Katsnelson, A. Tskhoidze, J. F. Lynch, and J. N. Moum, "Observation of sound focusing and defocusing due to propagating nonlinear internal waves," J. Acoust. Soc. Amer., vol. 124, no. 3, pp. EL66-EL72, Sep. 2008.

[24] H. A. DeFerrari, J. F. Lynch, and A. Newhall, "Temporal coherence of mode arrivals," J. Acoust. Soc. Amer., vol. 124, no. 3, pp. EL104-EL109, Sep. 2008.

[25] J. A. Flynn, J. A. Ritcey, D. Rouseff, and W. L. J. Fox, "Multichannel equalization by decision-directed passive phase conjugation: Experimental results," IEEE J. Ocean. Eng., vol. 29, no. 3, pp. 824-836, Jul. 2004.

[26] J. Gomes, A. Silva, and S. Jesus, "Adaptive spatial combining for passive time-reversed communications," J. Acoust. Soc. Amer., vol. 124, no. 2, pp. 1038-1053, Aug. 2008.

[27] H. C. Song and S. M. Kim, "Retrofocusing techniques in a waveguide for acoustic communications (L)," J. Acoust. Soc. Amer., vol. 121, no. 6, pp. 3277-3279, Jun. 2007.

[28] L. M. Brekhovskikh and Y. P. Lysanov, Fundamentals of Ocean Acoustics, 3rd ed. New York: Springer-Verlag, 2003, pp. 61-64.

[29] B. G. Katsnelson and V. G. Petnikov, Shallow-Water Acoustics. New York: Springer-Verlag, 2002, pp. 30-40.

[30] W. M. Carey, J. Doutt, R. B. Evans, and L. M. Dillman, "Shallow-water sound transmission measurements on the New Jersey Continent Shelf," IEEE J. Ocean. Eng., vol. 20, no. 4, pp. 321-331, Oct. 2000.

[31] J. Yang, D. Tang, and K. L. Williams, "Direct measurement of sediment sound speed using SAMS in SW06," J. Acoust. Soc. Amer., vol. 124, no. 3, pp. EL166-EL121, Sep. 2008.

[32] B. G. Katsnelson, V. Grigorev, and J. F. Lynch, "Intensity fluctuations of midfrequency sound signals passing through moving nonlinear internal waves," J. Acoust. Soc. Amer., vol. 124, no. 3, pp. EL78-EL84, 2008.

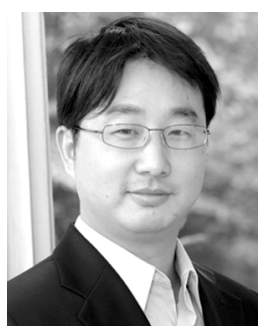

Aijun Song (S'02-M'05) received the Ph.D. degree in electrical engineering from the University of Delaware, Newark, in 2005.

From 2005 to 2008, he was a Postdoctoral Research Associate at the College of Earth, Ocean, and Environment (CEOE), University of Delaware. During this period, he was also an Office of Naval Research (ONR) Postdoctoral Fellow supported by the special research award from the Ocean Acoustics program. Since 2008, he has been an Assistant Research Professor of the Physical Ocean Science and Engineering program, University of Delaware. His general interests include underwater acoustic signal propagation, digital communication theory, and advanced signal processing in mobile radio frequency and underwater acoustic environments.

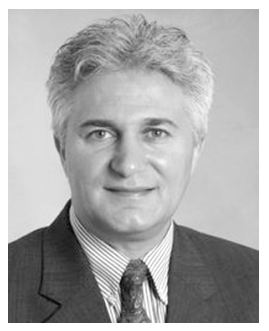

Mohsen Badiey (M'94) received the Ph.D. degree in applied marine physics and ocean engineering from the Rosentiel School of Marine and Atmospheric Science, University of Miami, Miami, FL, in 1988.

He was a Postdoctoral Fellow at the Port and Harbour Institute, Ministry of Transport, Tokyo, Japan, from 1989 through 1990, and worked on the water wave interaction with seafloor and seismic waves. In 1990, he became a faculty member at the College of Earth, Ocean, and Environment, University of Delaware, Newark, where he is presently a full Professor of Physical Ocean Science and Engineering. From 1992 to 1995, he was a Program Director and Scientific Officer directing the ocean acoustics program at the U.S. Office of Naval Research. His research interests are physics of sound and vibration, underwater acoustics in shallow-water regions, acoustical oceanography, underwater acoustic communications, seabed acoustics, and geophysics.

Dr. Badiey is a Fellow of the Acoustical Society of America. 


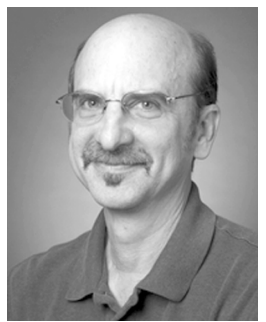

Arthur E. Newhall (M'07) received the B.S. degree in mathematics from the University of Maine at Orono, Orono, in 1985.

$\mathrm{He}$ is a Research Specialist at the Applied Ocean Physics and Engineering Department, Woods Hole Oceanographic Institution, Woods Hole, MA. His current interests include ocean acoustic propagation modeling, acoustical oceanography, software engineering, and music.

Mr. Newhall is a member of the Acoustical Society of America.

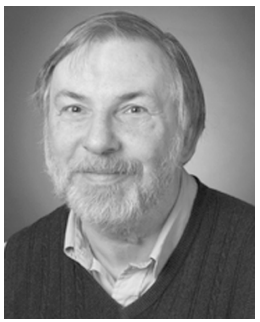

James F. Lynch (M'96-SM'02-F'05) was born in Jersey City, NJ, on June 3, 1950. He received the B.S. degree in physics from Stevens Institute of Technology, Hoboken, NJ, in 1972 and the Ph.D. degree in physics from the University of Texas at Austin, Austin, in 1978 .

$\mathrm{He}$ then worked for three years at the Applied Research Laboratories, University of Texas at Austin (ARL/UT) from 1978 to 1981, after which he joined the scientific staff at the Woods Hole Oceanographic Institution (WHOI), Woods Hole, MA. He has worked at WHOI since then, and currently holds the position of Senior Scientist in the Applied Ocean Physics and Engineering Department. His research specialty areas are ocean acoustics and acoustical oceanography, but he also greatly enjoys occasional forays into physical oceanography, marine geology, and marine biology.

Dr. Lynch is a Fellow of the Acoustical Society of America, the former Editor-in-Chief of the IEEE JOURNAL OF OCEANIC ENGINEERING and currently Chairman of the Applied Ocean Physics and Engineering Department at WHOI.

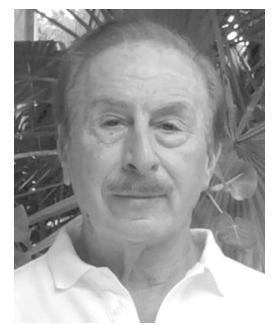

Harry A. DeFerrari received the Ph.D. degree in engineering from Catholic University, Washington, DC in 1966.

$\mathrm{He}$ has been a Professor of Applied Marine Physics at the Rosenstiel School of Marine and Atmospheric Science, University of Miami, Miami, FL, since 1967. He has supervised student research leading to over 35 graduate degrees. He has an active research program in ocean acoustics with emphasis on experiments at sea. Topics of interest include underwater acoustic signal processing, the application of codes sequences to active sonar and the coherence, fluctuations and predictability of long range propagation in shallow seas.

Prof. DeFerrari is a Fellow of the Acoustical Society of America and a recipient of the U.S. Navy's Distinguished Naval Educator Award.

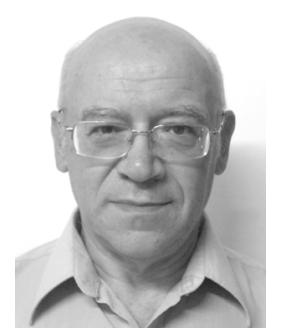

Boris G. Katsnelson received the B.S. degree in physics and the Ph.D. degree in theoretical physics from the Voronezh State University, Voronezh, Russia, in 1972 and 1976, respectively.

He was a Senior Scientist of the Chair of Theoretical Physics until 1988. Since 1989, he has been an Associate Professor of the Physics Department, Voronezh University. His research interests include wave propagation theory, underwater acoustics, and acoustical oceanography.

Dr. Katsnelson is a Fellow of the Acoustical Society of America and a member of the Russia Acoustical Society. 